\title{
Electrical standing waves in the HIFI HEB mixer amplifier chain
}

Ronan D. Higgins, Jacob W. Kooi

Ronan D. Higgins, Jacob W. Kooi, "Electrical standing waves in the HIFI HEB mixer amplifier chain," Proc. SPIE 7215, Terahertz Technology and Applications II, 72150L (10 February 2009); doi: 10.1117/12.811127

SPIE Event: SPIE OPTO: Integrated Optoelectronic Devices, 2009, San Jose, California, United States 


\title{
Electrical standing waves in the HIFI HEB mixer amplifier chain
}

\author{
Ronan D. Higgins ${ }^{a, b}$ and Jacob W. Kooi ${ }^{c}$ \\ ${ }^{a}$ National University of Ireland, Maynooth, Co. Kildare, Ireland \\ ${ }^{b}$ SRON Netherlands Institute for Space Research, PO Box 800, Groningen, the Netherlands \\ ${ }^{c}$ California Institute of Technology, MS 320-47, Pasadena, California 91125, U.S.A.
}

\section{ABSTRACT}

The Heterodyne Instrument for the Far-Infrared (HIFI) is one of three instruments to be launched aboard the Herschel Space Observatory (HSO) in 2009. HIFI will provide unprecedented spectral sensitivity and resolution between 490-1250 GHz and 1410-1910 GHz. In this paper, we report on the analysis of electrical standing waves that are present between the hot electron bolometer (HEB) heterodyne mixing element and the first low noise amplifier in the HIFI instrument. We show that the standing wave shape is not a standard sinusoid and difficult to remove from the resulting spectrum using standard fitting methods. We present a method to remove the standing waves based on data taken during the HIFI instrument level test, and anticipate the use of a similar calibration procedure in actual flight. Using the standing wave profile we obtain direct evidence of the complex IF output impedance of the HEB mixer.

Keywords: HIFI, Herschel, HEB mixer, standing wave, impedance

\section{INTRODUCTION}

The Heterodyne Instrument for the Far-Infrared (HIFI) is one of three instruments to be flown on the Herschel space observatory. ${ }^{1}$ HIFI will observe at high spectral resolution the complete range of frequencies from $480-$ $1250 \mathrm{GHz}$ and 1410-1910 GHz for two polarizations, divided over seven bands. This range of the electromagnetic spectrum is unique as it has never been comprehensively observed before. Observation at these wavelengths will answer numerous questions in the area of galaxy evolution and star and planet formation.

This range of the electromagnetic spectrum is also unique from an instrumentation point of view in that it falls between conventional operating limits of electronic and photonic devices. At millimeter and longer wavelengths low noise high electron mobility transistors (HEMTs) are the back bone of radio astronomy. However, due to the finite electron mobility of $I I I-V$ materials, their performance degrades rapidly as the wavelengths get shorter. At optical wavelengths down through to the mid-infrared, observations using quantum electronics are ideal as the higher photon energy allows efficient detection. At far infrared wavelengths the performance of quantum devices becomes problematic however due to the low photon energy. For this reason low energy gap superconducting materials are used for high sensitivity applications. In the case of low spectral resolution far-infrared observations superconducting transition-edge bolometers (TES) have achieved very high sensitivity. To achieve near quantum limited high spectral resolution observations, heterodyne techniques based on Superconducting-Insulator-Superconducting (SIS) tunnel junctions and Hot Electron Bolometers (HEBs) need to be employed.

The heterodyne technique works by multiplying (mixing) the incoming sky signal with a monochromatic local signal (the Local Oscillator, LO) by means of a highly non-linear mixing element, sensitive to the beat term between the two signals. The HIFI instrument uses this technique to down-convert an upper and lower band of terahertz radiation to the microwave frequency domain where it can be sampled and recorded using conventional electronics. At the output of the mixer the beat term between the LO frequency and the signal frequency (also

Further author information: (Send correspondence to R.D.H)

R.D.H.: E-mail: daniel.r.higgins@nuim.ie, Telephone: +353-1-708-3641

J.W.K.: E-mail: kooi@submm.caltech.edu, Telephone: +1-626-395-4286

Terahertz Technology and Applications II, edited by Kurt J. Linden, Laurence P. Sadwick, Créidhe M. M. O'Sullivan, Proc. of SPIE Vol. 7215, 72150L · C 2009 SPIE · CCC code: 0277-786X/09/\$18 · doi: 10.1117/12.811127 
known as the intermediate frequency or IF) is then typically fed to a spectrometer where the spectral information is analyzed. In this way very high resolution observations (defined as $R=\nu / \Delta \nu \geq 10^{6}-10^{7}$ ) may be obtained. After down conversion the double sideband spectra are fed to the backend spectrometers*. To deconvolve the signals in the upper and lower sideband a deconvolution algorithm ${ }^{2}$ will be used in the instrument data processing pipeline.

The HIFI instrument use two types of mixing elements. SIS mixers are used from $480-1250 \mathrm{GHz}$ (bands 1-5) and HEB mixers from 1410-1910 GHz (bands 6-7). Both mixers use superconducting principles to mix the incoming terahertz sky signal with the LO signal, albeit with completely different mechanisms.

The HEB mixer technology used in mixer bands 6-7 was first proposed in the early 1970's ${ }^{3}$ but then superseded by SIS mixer technology in the 1980's. The range of frequencies of SIS mixers are however limited by the energy gap of the superconducting material (above this frequency absorption loss in the superconducting film due to the breaking of Cooper pairs increases dramatically). SIS mixer techniques are a key technology of submillimeter spectroscopy and have been used extensively in ground based telescopes for the last twenty years. ${ }^{4,5}$ Due to the energy gap limitation of SIS mixers, HEB mixers are used above $\sim 1.25 \mathrm{GHz}$. The two mixer types were envisaged to produce a common $4-8 \mathrm{GHz}$ bandwidth, and hence use an identical IF chain and output to the same backend spectrometers. Due to development issues with the HEB mixers, the original 4-8 GHz IF bandwidth needed to be reduced to $2.4-4.8 \mathrm{GHz}$. The IF chain configuration was subsequently changed to match the new mixer bandwidth, however without the use of an isolator between the mixer and first low noise amplifier (LNA), as shown in Fig. 1. This was due to the lack of availability of an appropriate (space qualified) isolator for the new bandwidth. As a result of this configuration change the impedance mismatch between the HEB mixer and the first cryogenically cooled LNA becomes very important.

This paper will discuss the resulting power reflection (standing waves) between the HEB mixer and the first low noise amplifier. Section 3 describes the problem of HEB bandwidth and the missing isolators in the IF chain. Section 4 details how the standing wave affects in-flight observations and how a standard fitting approach fails to remove, to a satisfactory level, the effect. Section 5 describes the current HEB impedance mixer theory and how, using this theory, a fit to the IF standing wave is possible. This approach facilitates baseline correction but also allows the extraction of physically relevant device parameters, such as the electron temperature in the hot electron bolometer bridge. Finally, section 6 presents the proposed solution of using the calibration data taken during the HIFI instrument level tests of 2007 to remove the IF standing wave.

\section{HEB IF CHAIN}

The IF chain is used to amplify the mixer output signal with as little added noise as possible, and provide a link to the back-end spectrometers. The IF chain passband is designed to transmit the signal equally over the entire IF bandwidth while minimizing reflection loss between the individual components. The latter is typically accomplished by careful matching of the impedance between components. To further reduce reflections between the IF components fixed $3 \mathrm{~dB}$ attenuators have been added at certain crucial locations, as indicated in Fig. 1

When HIFI was originally proposed in 1998 it was envisaged that the two bands above $1250 \mathrm{GHz}$ would use HEB mixer technology. At the time two mixer technologies were proposed: A baseline of diffusion-cooled $\mathrm{Nb}$ HEBs and a goal of diffusion-cooled Al HEBs. Diffusion cooled HEBs were chosen over phonon cooled NbN HEBs on the basis of potentially superior sensitivity and higher IF bandwidth. It was argued that the diffusion-cooled technology could reach the higher IF bandwidth simply by reducing the length of the superconducting bolometer element whereas the phonon-cooled technology's maximum IF bandwidth is more dependent on intrinsic material properties, and thus not easily extendable. Initial results proved promising ${ }^{6}$ but the diffusion cooled HEB didn't produce repeatable results for subsequent mixer batches. In 2002 the decision was made to switch from the diffusion cooled HEBs to the (by then) proven phonon cooled HEB mixer technology. ${ }^{7}$ This required a redesign of the IF chain to match the reduced bandwidth. ${ }^{8}$

As we have stated, in the original IF chain design it was envisaged that the seven HIFI bands would have the same IF bandwidth $(4-8 \mathrm{GHz})$ and therefore have a common IF chain design. However due to the change

${ }^{*}$ HIFI has four backend spectrometers, 2 Wide Band Spectrometers (WBS) and and 2 High Resolution Spectrometers (HRS), which observe the $\mathrm{H}$ and $\mathrm{V}$ polarization components of the signal. 


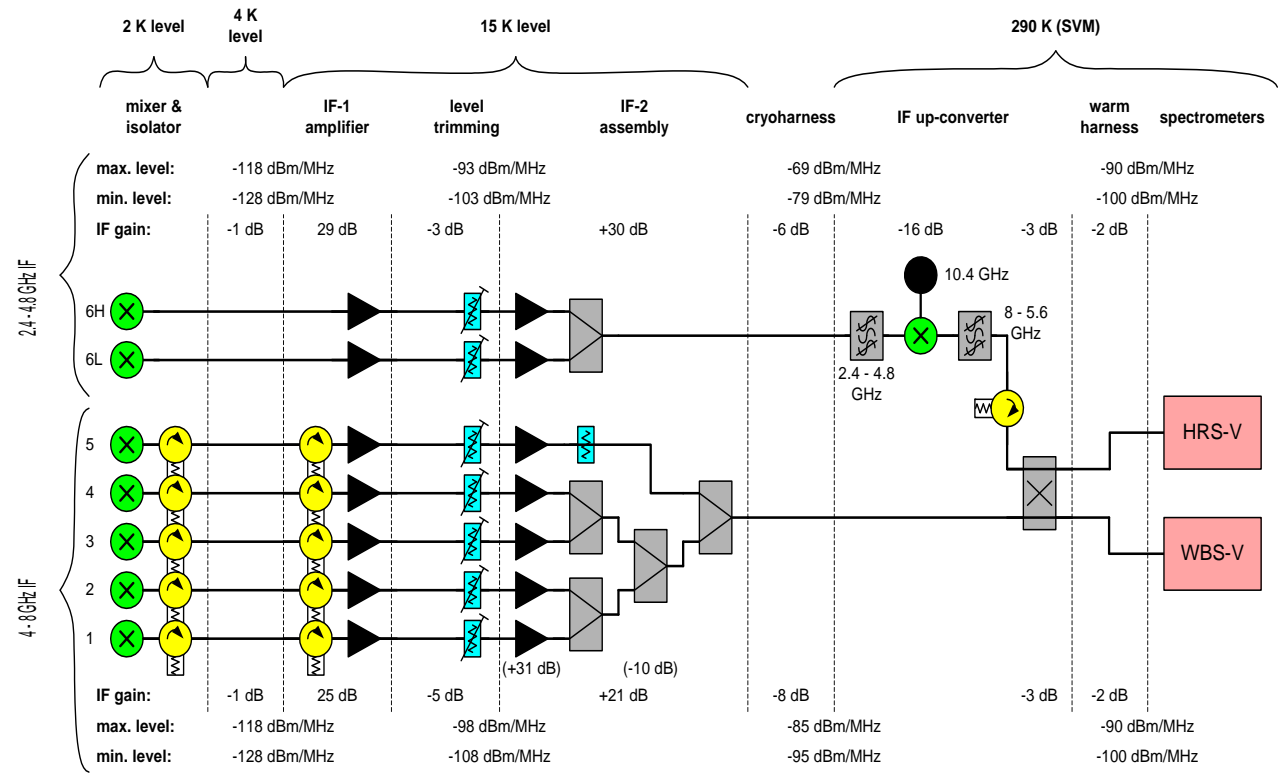

Figure 1. Block diagram of the HIFI signal chain showing all components for one polarization ${ }^{1}$

in HEB bandwidth a new IF chain design was necessary for bands $6 \& 7$. The first and second amplifiers were redesigned to accommodate the new bandwidth and a $10.4 \mathrm{GHz}$ oscillator was added after the second amplifier to up-convert the $2.4-4.8 \mathrm{GHz}$ bandwidth to a spectrometer compatible bandwidth of 8-5.6 GHz. Due to time and mass/volume constraints it was not possible to develop and procure a new cryogenic isolator, and it was decided to remove the isolators from the HEB IF chain. Initially it was believed that the IF chain could be designed to work without isolators due to the supposed benign $(50 \Omega)$ output impedance of the HEB mixer, but this proved to be false. The first IF design had noise temperatures in excess of $10,000 \mathrm{~K}$ and a large $300 \mathrm{MHz}$ ripple due to power reflections between the mixer and first LNA. After a careful analyzes of the actual HEB mixer IF output impedance, ${ }^{9}$ a redesign of the HEB mixer unit IF board and matching circuit was judged needed. Subsequent measurements showed that the large excess noise was eliminated, however there remained (not unexpected) a significant IF standing wave. The final IF chain design is shown in Fig. 1.

\section{STANDING WAVE PROFILE}

Optical standing waves are a common feature in submm instruments. They occur when the signal wavelength is comparable with the instrument dimensions. Standing waves occur between two surfaces when a fraction of the incoming radiation is reflected back along the path of the incoming radiation, and the reflected electromagnetic wave interferes with the incoming signal. Depending on the relative phase of the incoming and reflected signal, this phenomena can either add or subtract the total signal detected. It thus produces a sinusoid on the backend spectrometer baseline, the period, $P$, of this sinusoid is a function of the distance, $d$, between the two reflecting surfaces and the speed of light, $c$, in the propagation medium, see Eq. 1. The amplitude is a function of the beam coupling between the two surfaces. ${ }^{10}$

$$
P=c / 2 d
$$

Standing wave removal is a common feature in the data pipelines of astronomical instruments. The HIFI instrument pipeline has a dedicated standing wave removal tool based on the heritage of previous ESA missions. ${ }^{11}$ These tools are effective at removing the optical standing waves in the HIFI data but have proven only partially 

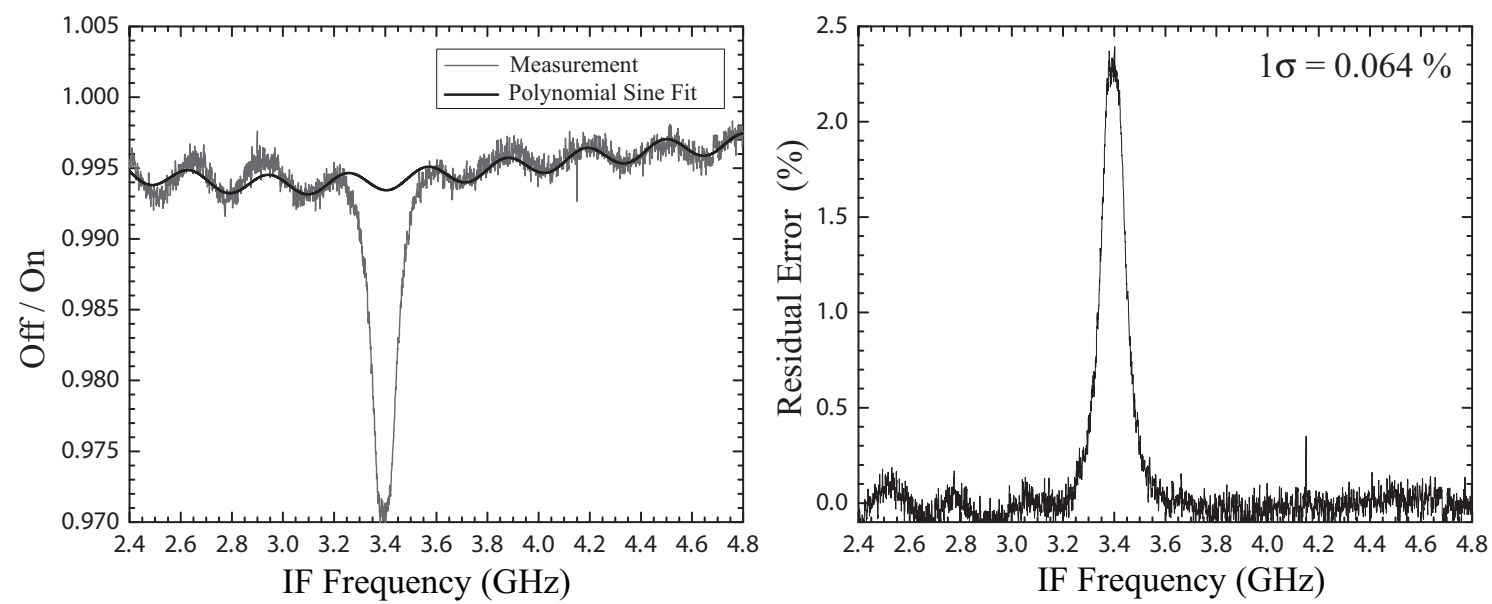

Figure 2. Standard fitting approach using a polynomial fit to correct for baseline slope and a sine fitting to fit the sinusoid pattern. This plot shows the ${ }^{12} \mathrm{CO}(15-14)$ line at $1726.602 \mathrm{GHz}$ for a LO frequency of $1730.00475 \mathrm{GHz}$. The line is observed in the lower side band, at the IF frequency of $\sim 3.403 \mathrm{GHz}$ corresponding to the beat frequency between the LO frequency and the line frequency. This data was taken during the HIFI gas cell tests and shows an Off/On observation.

effective at removing the intermediate frequency electrical standing waves seen in the HEB bands, see Fig. 2. Unlike the electrical (IF) standing wave, optical standing waves have a fixed period across the IF bandwidth. Depending on whether the LO and sky signals are coupled via a beam splitter (bands 1,2 \& 5) or diplexer (bands $3,4 \& 6,7)$ they can have a fixed or variable amplitude across the IF bandwidth. ${ }^{12}$

The electrical standing waves seen in bands $6 \& 7$ are more complicated. They occur due to reflections between the HEB mixer and first LNA. Unlike optical reflection surfaces, the reflection properties (electrical impedance) of these components vary significantly over the IF bandwidth. Additionally, the HEB mixer has a significant complex impedance (section 5) which results in a saw-tooth like distortion to the standing wave shape and thus difficult to fit using standard routines. As demonstrated in Fig. 2, it is possible to fit the standing wave for a portion of the IF band using a fixed amplitude and period sinusoid but this is not a sufficient correction for astronomers who may want to use the entire passband.

A more sophisticated fitting approach using a variable period sinusoid coupled with a polynomial amplitude distribution was able to match the saw-tooth profile, but again was only effective for $\sim 1 \mathrm{GHz}$ bands of the IF. In order to fully fit the standing wave profile, an understanding of the reflecting components is thus required. The impedance of the LNA is available as part of component acceptance testing. However, the impedance of the HEB mixer is inherently difficult to measure due to its cryogenic operating temperature and unreliability of calibration loads at these temperatures. The next section will elaborate on the source of the standing wave, the underlying physics of HEB impedance and the resulting standing wave fit produced by an IF chain electrical model.

\section{HEB IMPEDANCE MODEL}

During a typical astronomical observation run the HEB input beam is switched between the desired signal source and a (blank-sky) reference beam. In an ideal system all components in the IF system are stable over a time period greater than the actual observation. Unfortunately, HEB mixers are far from ideal, and small power (temperature) changes at the mixer input result in a change in the HEB mixer IF output impedance. These changes may for example be due to a change in the incident local oscillator power, mixer bias voltage, and/or continuum level input temperature. To make matters worse, changes in voltage reflections at the mixer IF output port cause a small change in the hot electron bolometer distributed temperature profile, ${ }^{13}$ thus providing a positive feedback mechanism. This is known as electro-thermal feedback. These small changes in impedance result in a non-perfect cancellation of instrument effects and the observer is left with a distorted baseline. Since 


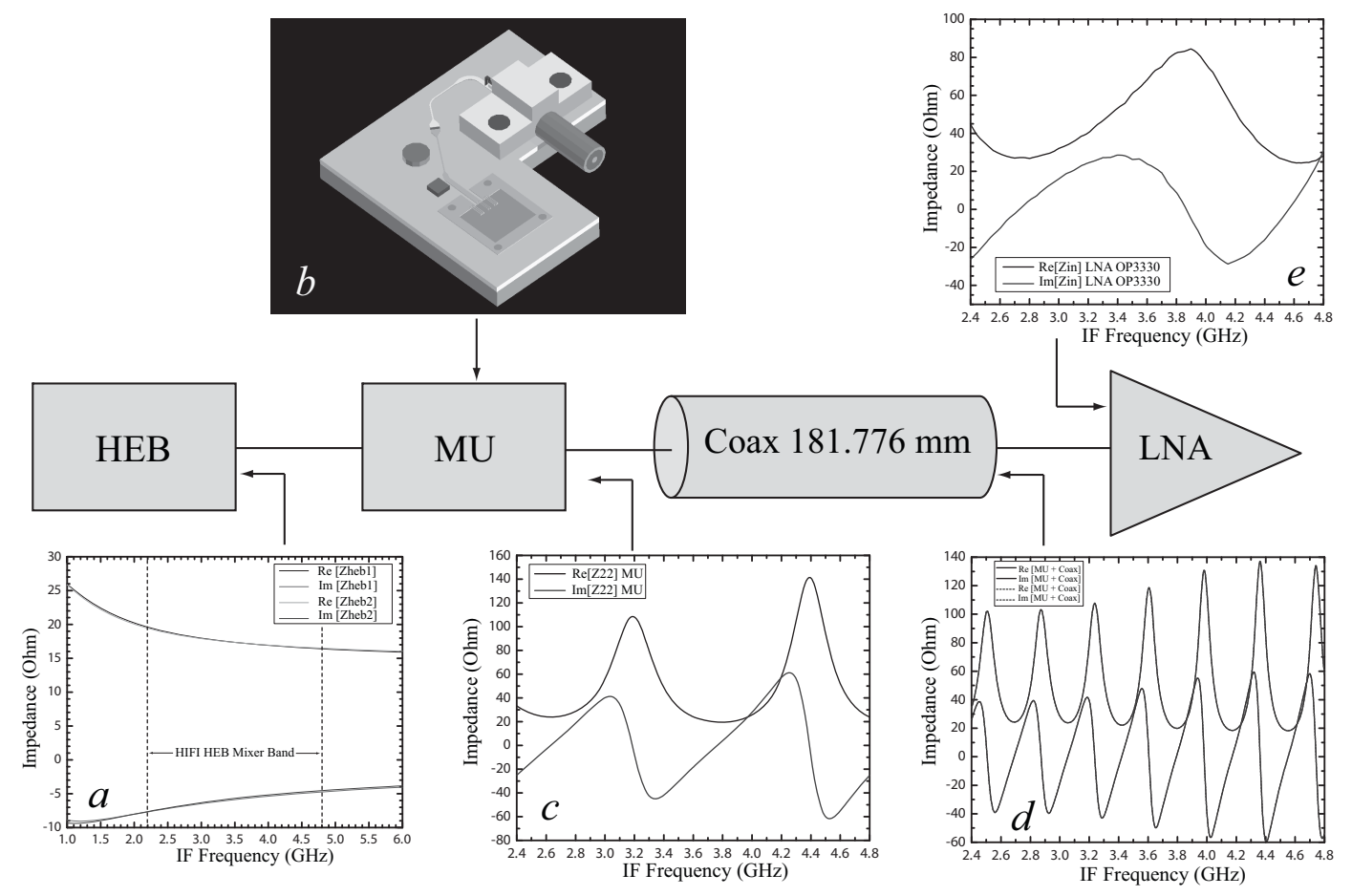

Figure 3. The HIFI instrument band 6 \& 7 IF chain. a) The modeled real and complex part of the HEB mixer IF output impedance (see text for details). This impedance is then transformed by the 'mixer unit', depicted in $b$, to yield an IF impedance as shown in panel $c$. The complex nature of space qualified hardware made it impossible to alter the mixer unit physical layout, hence the non-ideal impedance (note that the locus is around $50 \Omega$ ). After passing through a $\sim 182 \mathrm{~mm}$ (effective length obtained from a fit to the data) $50 \Omega$ semi-ridged coax cable the IF impedance is then transformed to that shown in panel $d$. The IF chain having no isolator connects directly to the first LNA, whose measured input impedance is shown in panel $e$. The result is a $\sim 300 \mathrm{MHz}$ standing wave in the IF passband (Fig. 5).

the LNA is kept in a fixed state during observations the amplitude of the residual standing wave is a measure of how far the mixer impedance has drifted between two observations.

To understand how the frequency dependent IF output impedance of the HEB mixer modulates the standing wave consider the model of the HIFI IF chain shown in Fig. 3. As stated in section 3, due to the complex nature of space qualified hardware it was not possible to change the layout of the mixer unit, hence the somewhat non-optimal design.

To better comprehend the nature and frequency dependence of the HEB mixer output impedance we follow the analyzes of Nebosis and Kooi et al..$^{9,14}$ A HEB mixer operates in principle very close to the critical temperature of the superconducting material used. For NbN films this is around $10 \mathrm{~K}$. Both LO power and bias are used to bring the HEB bridge temperature close to the ideal transition, or operating temperature. Since the local oscillator frequency is well above the energy gap of the superconducting film, heating in the film may be considered uniform. To describe the thermal balance in the HEB bridge we have to consider the phonon-electron interaction time $\left(\tau_{e p h}\right)$, the phonon escape time $\left(\tau_{e s c}\right)$ to the lattice (substrate), and the temperature of the quasi-particles $\left(T_{e}\right)$. The electron-electron interaction time is assumed must faster then the above mentioned time constants.

Diffusion loss via the normal conducting $(\mathrm{Au})$ contact pads may be neglected for the predominantly 'phonon' cooled HEB mixer we consider ourselves with.

Using the two temperature electron cooling model with associated heat balance equations of a thin 2D film as described by Perrin-Vanneste (PV), ${ }^{15}$ and expanded upon by Nebosis, Semenov, Gousev, and Renk (NSGR), ${ }^{14}$ while making the assumption that at IF frequencies the temporal response is predominantly governed by the electron temperature dependent change in resistance $\left(\partial R / \partial T_{e}\right)$ we obtain the complex IF impedance 


$$
Z(\omega)=R_{0} \cdot \frac{\Psi(\omega)+C}{\Psi(\omega)-C} .
$$

In the above equation $\Psi(\omega)$ represents the time dependent modulation of the electron temperature and is defined by three time constants, $\tau_{1}, \tau_{2}, \tau_{3}$

$$
\Psi(\omega)=\frac{\left(1+i \omega \tau_{1}\right)\left(1+i \omega \tau_{2}\right)}{\left(1+i \omega \tau_{3}\right)} .
$$

$C$ is the self heating parameter and can be described as

$$
C=\frac{I^{2}}{V} \frac{\partial R}{\partial T_{e}}\left(\frac{\tau_{e p h}}{c_{e}}+\frac{\tau_{e s c}}{c_{p h}}\right)
$$

In the above equations $R_{0}$ is the dc resistance at the operating point of the mixer $(\sim 15 \Omega)$. The differential resistance at the operating point, $\partial R / \partial V$, is under normal bias conditions $\sim 60 \Omega .^{7} c_{e}$ and $c_{p h}$ are the respective heat capacities of the electrons and phonons. The time constants $\tau_{1}, \tau_{2}, \tau_{3}$ depend on $\tau_{e p h}, \tau_{e s c}$, $c_{e} / c_{p h}$ which are derived from the empirical relationship that for thin $\mathrm{NbN}$ films $\left\langle\tau_{\text {esc }}\right\rangle \sim 10.5 d(\mathrm{ps} / \mathrm{nm})^{16},{ }^{17}$ $\tau_{e p h} \approx 500 T^{-1.6}(\mathrm{ps} \cdot \mathrm{K})^{18}$ and that $c_{e} / c_{p h} \approx 18.77 T^{-2} .{ }^{17}$ In the previous equation $d$ is the thickness of the $(\mathrm{NbN})$ film in the HEB bridge. In Fig. 4 we show $\tau_{1}, \tau_{2}, \tau_{3}$ as a function of electron temperature. From a fit of the model against the measurement we obtain an electron temperature of $\sim 10 \mathrm{~K}$, consistent with the outlined theory.

$$
\tau_{1}^{-1}, \tau_{2}^{-1}=\frac{\Omega}{2}\left(1 \mp \sqrt{1-\frac{4 \tau_{e p h}^{-1} \tau_{e s c}^{-1}}{\Omega^{2}}}\right),
$$

with

$$
\Omega=\left(1+\frac{c_{e}}{c_{p h}}\right) \cdot \tau_{e p h}^{-1}+\tau_{e s c}^{-1}
$$

and

$$
\tau_{3}^{-1}=\frac{c_{e}}{c_{p h}} \tau_{e p h}^{-1}+\tau_{e s c}^{-1} .
$$

As the local oscillator power or bias voltage changes, ${ }^{9}$ the electron temperature in the bridge changes, effecting the physical parameters and thereby $\tau_{1}, \tau_{2}, \tau_{3}$. This is illustrated in Fig. 4 . The change in IF output impedance is small, but not negligible (Fig. 3a).

To calculate the effect of the HEB mixer IF output impedance on the instrument we first transform the derived HEB impedance to the mixer unit (MU) output. This is accomplished via a S-parameter model (Fig. 3) derived from electromagnetic finite element analysis. ${ }^{19}$ In this model the IF bond wires are included. Next we transform the mixer unit output impedance via an effective $181.776 \mathrm{~mm}$ (derived from a fit against the data) electrical length coaxial cable to the input of the first low noise amplifier. Using a linear circuit simulator ${ }^{20}$ we calculate the overall gain of the system. If we next allow the electron temperature of the bridge to vary to mimic an actual 'off' position observation we than obtain the normalized $O f f / O n$ spectrum with the residual standing wave. By varying $\tau_{e p h}, \tau_{e s c}$, and $c_{e} / c_{p h}$ in our HEB model, we can 'fit' the measurement and thus extract information on the actual electron temperature of the bridge. The thus obtained parameters are shown in Table 1. The quality of the depicted fit provides a nice confirmation of the 'NSGR' model. ${ }^{14}$ It does however also show that there are some lower order residuals present that cannot be accounted for by the model, thereby limiting the accuracy of the $O f f / O n$ correction. To improve the quality and numerical calculation overhead of the IF standing wave removal we now introduce a new technique as outlined in Section 6 . 


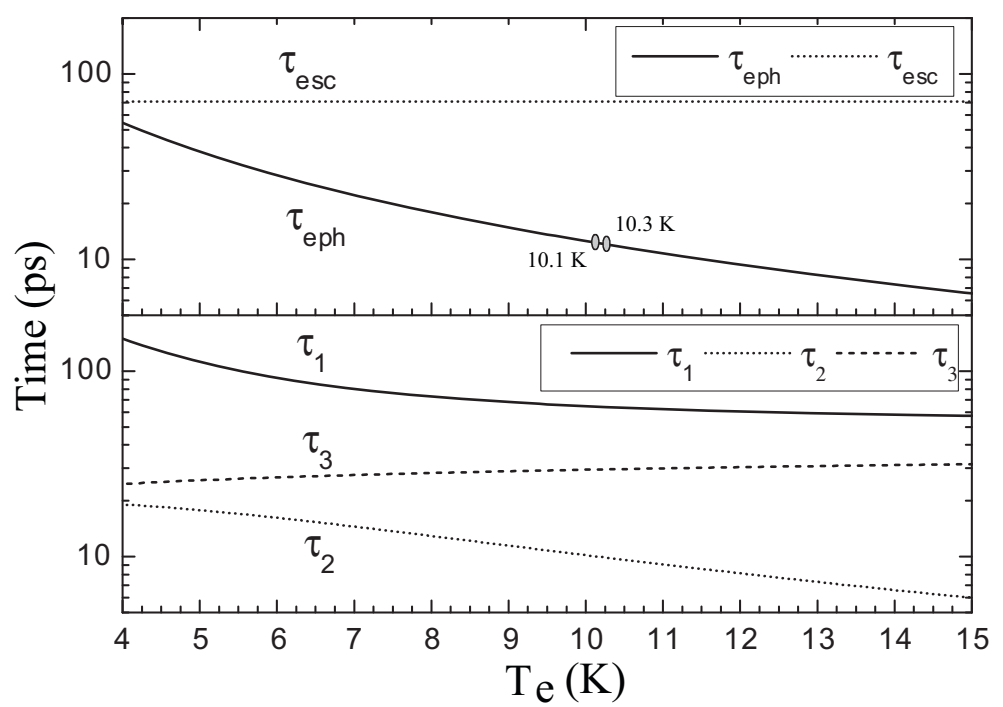

Figure 4. Discussed time constants and their electron temperature relationship. The $\mathrm{NbN} \tau_{\text {eph }}, \tau_{\text {esc }}$, and the heat capacity ratio $c_{e} / c_{p h}$ (not shown) are obtained from literature and serve to constrain the impedance and mixer gain models. From a fit of the model against measurement (Fig 3) we derive a $6.4 \mathrm{~nm}$ thick NbN film. The electron temperature of the 'on' and 'off' source signals are $10.1 \& 10.3 \mathrm{~K}$ respectively. The redistribution of the electron temperature is due to a small change in LO power (optical standing wave, LO power drift) and/or bias change. This results in a slight change in the mixer output impedance (Fig. 3a), and a non-prefect 'on' - 'off' subtraction, and hence a residual IF standing wave in thus obtained spectrum.

Table 1. Derived HEB mixer parameters for the 'On' and 'Off' source observations as based on the outlined impedance model. Units of $R_{o}$ is in $\Omega, \tau_{e s c}$ and $\tau_{e p h}$ in ps, and $T_{e}$ in Kelvin.

\begin{tabular}{lcccccc}
\hline \hline mode & $R_{o}$ & $C$ & $\tau_{e s c}$ & $\tau_{e p h}$ & $c_{e} / c_{p h}$ & $T_{e}$ \\
\hline On & 14.394 & 0.3995 & 71.701 & 12.108 & 0.1978 & 10.1 \\
Off & 14.715 & 0.4007 & 73.397 & 12.314 & 0.2297 & 10.3 \\
\hline
\end{tabular}
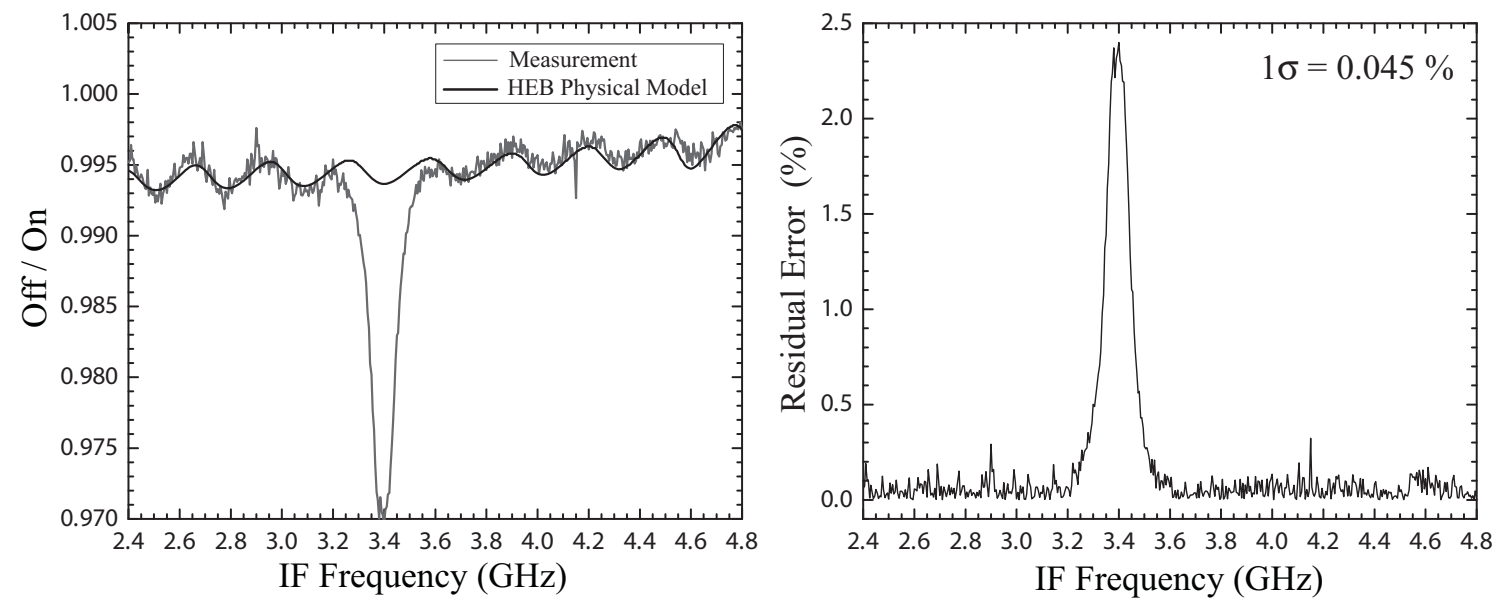

Figure 5. Left) Measured and modeled Off/On IF standing wave. The impedance model was fit against the measurement, from which the physical constants $\tau_{e p h}, \tau_{e s c}$, and $c_{e} / c_{p h}$ are estimated. From the empirical relationship to the electron temperature in literature we obtain an estimate of the actual electron temperature during the (gas-cell) observation. The thus obtained parameters are compiled in Table 1. Right) Residual error of the measurement minus the model. The region around the absorption line has been omitted during the fitting routine. 


\section{STANDING WAVE SOLUTION}
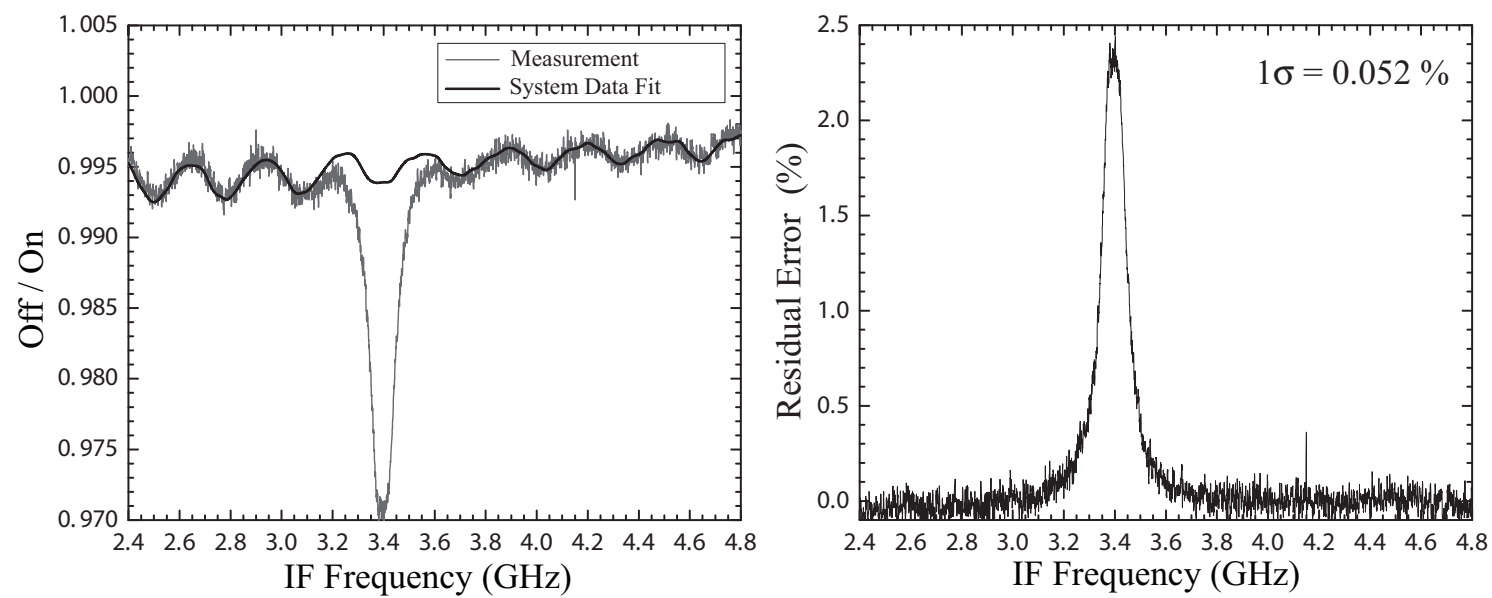

Figure 6. ${ }^{12} \mathrm{CO}(15-14)$ data fitted with a smoothed mixer current equivalent spectra taken from the HIFI stability test data.

We have shown in section 5 that a fitting approach based on the fundamental physics of the HEB mixer coupled with a model of the coaxial cable and the first LNA provides a good first order fit to the standing wave profile. However this approach is computationally expensive given that four free parameters are used for each calibration phase. In the example spectra shown we have used a 2 phase calibration, Off/On, to remove system artifacts from the spectra. For HIFI operation the observed spectra will be temperature calibrated using a four phase calibration routine incorporating the HIFI internal hot and cold loads. The four phase calibration equation is as follows:

$$
\frac{O n-O f f}{\text { Hot }- \text { Cold }}
$$

Fitting the standing wave for a four phase calibration using the HEB model becomes problematic as the amount of parameters increases to 16. Additionally, HIFI will take multiple spectra during routine operation, which are calibrated individually using equation 8. This further increases the computation time for removal of the HEB standing wave. For a one minute integration time and a nominal WBS mode of 4 second readout this would generate up to 15 calibrated spectra which would be computationally extensive using the method proposed in section 5 .

As part of our investigation into the standing wave effect in the HEB bands we noticed a correlation between the mixer current and the amplitude of the standing wave present in the spectra. It was seen that the greater the difference in mixer current, $\Delta I_{m i x}$, between two calibration phases the larger the residual standing wave present in the calibrated spectra. This correlation between $\Delta I_{m i x}$ and the residual standing wave was expected since $I_{m i x}$ can be taken as a measure of the mixer impedance given that the mixer bias voltage, $V_{0}$, is a fixed parameter.

Furthermore, it was seen that there was a remarkable stability in the standing wave profile for a given $\Delta I_{m i x}$ between two calibration phases at a given $I_{m i x}$ when compared to equivalent spectra taken at different times and LO frequencies for the same HEB mixer unit. Using this standing wave stability we devised a method to match the gas cell spectra with equivalent test spectra obtained from the HIFI instrument level test (ILT) local oscillator warm up characterization. The ILT LO warm up data provide a large sample of spectra at different mixer currents at the same LO frequency. Using this data we matched the mixer current seen in the gas cell phases with an equivalent test spectra (also based on current), the results of which are shown in figure 6 .

This approach has proved very effective as a method to remove the (IF) electrical standing wave from the spectra of HEB band observations. Significant, this method is easier to implement than the HEB impedance 
model method presented in section 5 and less computationally extensive. This method will be included as a module in the HIFI instrument pipeline. Further tests are to be undertaken as part of the commissioning phase of HIFI in order to generate a complete catalog of standing wave spectra for a range of mixer currents for each of the four HEBs present in the HIFI instrument.

\section{CONCLUSIONS}

In this paper, we presented a method to fit the electrical standing waves present in the HEB mixer IF chain of the Herschel HIFI spectrometer. We have shown that the IF standing wave can be removed by using equivalent mixer current spectra taken from calibration data obtained during LO warm up. This method will be included in the HIFI instrument pipeline.

In addition, we have shown that the HEB impedance model generates a good first order fit to the standing wave profile. The fit parameters returns information about the conditions at the mixer bridge which have previously not been directly observed. This fit supports the HEB impedance model presented by Nebosis and Kooi et al. ${ }^{9,14}$

\section{Acknowledgements}

The authors thank the gas cell team for providing the data presented in this paper and Bertrand Delforge for generating the gascell database and the tools to access it. Additionally the authors thank David Teyssier, Pourya Khosropanah, Willem Jellema, Gert de Lange and Pieter Dieleman for their insights about HIFI and the HEB IF chain. The authors would like to acknowledge the support of Enterprise Ireland (Prodex).

\section{REFERENCES}

[1] de Graauw, T., Whyborn, N., Helmich, F., Dieleman, P., Roelfsema, P., Caux, E., Phillips, T., Stutzki, J., Beintema, D., Benz, A., Biver, N., Boogert, A., Boulanger, F., Cherednichenko, S., Coeur-Joly, O., Comito, C., Dartois, E., de Jonge, A., de Lange, G., Delorme, I., DiGiorgio, A., Dubbeldam, L., Edwards, K., Fich, M., Güsten, R., Herpin, F., Honingh, N., Huisman, R., Jacobs, H., Jellema, W., Kawamura, J., Kester, D., Klapwijk, T., Klein, T., Kooi, J., Krieg, J.-M., Kramer, C., Kruizenga, B., Laauwen, W., Larsson, B., Leinz, C., Liseau, R., Lord, S., Luinge, W., Marston, A., Merkel, H., Moreno, R., Morris, P., Murphy, A., Naber, A., Planesas, P., Martin-Pintado, J., Olberg, M., Orleanski, P., Ossenkopf, V., Pearson, J., Perault, M., Phillip, S., Rataj, M., Ravera, L., Saraceno, P., Schieder, R., Schmuelling, F., Szczerba, R., Shipman, R., Teyssier, D., Vastel, C., Visser, H., Wildeman, K., Wafelbakker, K., Ward, J., Higgins, R., Aarts, H., Tielens, X., and Zaal, P., "The Herschel-Heterodyne Instrument for the Far-Infrared (HIFI):instrument and pre-launch testing," Space Telescopes and Instrumentation 2008: Optical, Infrared, and Millimeter 7010(1), 701004, SPIE (2008).

[2] Comito, C. and Schilke, P., "Reconstructing reality: Strategies for sideband deconvolution," A\&A 395, 357-371 (Nov. 2002).

[3] Phillips, T. G. and Jefferts, K. B., "A low temperature bolometer heterodyne receiver for millimeter wave astronomy." Review of Scientific Instruments 44, 1009-1014 (1973).

[4] Zmuidzinas, J. and Richards, P., "Superconducting detectors and mixers for millimeter and submillimeter astrophysics," Proceedings of the IEEE 92, 1597-1616 (Oct. 2004).

[5] Kooi, J. W., Kovács, A., Sumner, M. C., Chattopadhyay, G., Ceria, R., Miller, D., Bumble, B., Leduc, H. G., Stern, J. A., and Phillips, T. G., "A 275-425 GHz Tunerless Waveguide Receiver Based on AlN-Barrier SIS Technology," IEEE Transactions on Microwave Theory Techniques 55, 2086-2096 (Oct. 2007).

[6] Kawamura, J., Bumble, B., Harding, D. G., McGrath, W. R., Focardi, P., and LeDuc, R., "1.8 THz superconductive hot-electron bolometer mixer for Herschel," in [Society of Photo-Optical Instrumentation Engineers (SPIE) Conference Series], Phillips, T. G. and Zmuidzinas, J., eds., Society of Photo-Optical Instrumentation Engineers (SPIE) Conference Series 4855, 355-360 (Feb. 2003).

[7] Cherednichenko, S., Drakinskiy, V., Berg, T., Khosropanah, P., and Kollberg, E., "Hot-electron bolometer terahertz mixers for the Herschel Space Observatory," Review of Scientific Instruments 79, 034501-+ (Mar. 2008). 
[8] Whyborn, N., "Band 6 IF change impact assessment," tech. rep., SRON (2002).

[9] Kooi, J. W., Baselmans, J. J. A., Hajenius, M., Gao, J. R., Klapwijk, T. M., Dieleman, P., Baryshev, A., and de Lange, G., "IF impedance and mixer gain of $\mathrm{NbN}$ hot electron bolometers," Journal of Applied Physics 101(4), 044511 (2007).

[10] Trappe, N., Finn, T., Murphy, J. A., Withington, S., and Jellema, W., "Analysis of standing waves in submillimeter-wave optics," in [Society of Photo-Optical Instrumentation Engineers (SPIE) Conference Series], Hwu, R. J. and Linden, K. J., eds., Society of Photo-Optical Instrumentation Engineers (SPIE) Conference Series 6120, 107-116 (Mar. 2006).

[11] Kester, D. J. M., Beintema, D. A., and Lutz, D., "SWS Fringes and Models," in [The Calibration Legacy of the ISO Mission], Metcalfe, L., Salama, A., Peschke, S. B., and Kessler, M. F., eds., ESA Special Publication 481, 375-+ (2003).

[12] Siebertz, O., Honingh, C., Tils, T., Gal, C., and Olbrich, M., "The impact of standing waves in the LO path of a heterodyne receiver," IEEE Transactions on Microwave Theory Techniques 50(1) (2002).

[13] Klapwijk, T. M., Barends, R., Gao, J., Hajenius, M., and Baselmans, J. J. A., "Improved superconducting hot-electron bolometer devices for the THz range," in [Society of Photo-Optical Instrumentation Engineers (SPIE) Conference Series], Bradford, C. M., Ade, P. A. R., Aguirre, J. E., Bock, J. J., Dragovan, M., Duband, L., Earle, L., Glenn, J., Matsuhara, H., Naylor, B. J., Nguyen, H. T., Yun, M., and Zmuidzinas, J., eds., Society of Photo-Optical Instrumentation Engineers (SPIE) Conference Series 5498, 129-139 (Oct. 2004).

[14] Nebosis, R. S., Semenov, A. D., Gousev, Y. P., and Renk, K. F., "Rigorous Analysis of a Superconducting Hot-Electron Bolometer Mixer: Theory and Comparison with Experiment," Proc. "rth Int. Symp. on Space Terahertz Tech. , 601-613 (March 1996).

[15] Perrin, N. and Vanneste, C. , "Dynamic behaviour of a superconductor under time-dependent external excitation," Journal de Physique 48(8), 1311-1316 (1987).

[16] Kaplan, S. B., "Acoustic matching of superconducting films to substrates," Journal of Low Temperature Physics 37, 343-365 (Nov. 1979).

[17] Semenov, A. D., Nebosis, R. S., Gousev, Y. P., Heusinger, M. A., and Renk, K. F., "Analysis of the nonequilibrium photoresponse of superconducting films to pulsed radiation by use of a two-temperature model," Phys. Rev. B 52, 581-590 (July 1995).

[18] Gousev, Y. P., Gol'Tsman, G. N., Semenov, A. D., Gershenzon, E. M., Nebosis, R. S., Heusinger, M. A., and Renk, K. F., "Broadband ultrafast superconducting NbN detector for electromagnetic radiation," Journal of Applied Physics 75, 3695-3697 (Apr. 1994).

[19] "Ansoft Corporation, Pittsburgh, PA 15219, USA.," (November 2008).

[20] "Microwave office, Applied Wave Research Inc. El Segundo, CA 90245, USA.," (November 2008). 